\title{
3 Research Square

\section{A Critical Analysis of the Multi-Focus Images Fusion Based on Discrete Wavelet Transform and Computer Vision}

Gebeyehu Belay Gebremeskel ( $\sim$ ge.be09@yahoo.com )

Bahir Dar University Institute of Technology https://orcid.org/0000-0002-6101-6204

\section{Research Article}

Keywords: multi-focus image, wavelet transformation, wavelet basis function, wavelet decomposing level, region contract, computer vision,

Posted Date: December 20th, 2021

DOI: https://doi.org/10.21203/rs.3.rs-1176759/v1

License: (c) (1) This work is licensed under a Creative Commons Attribution 4.0 International License.

Read Full License 


\title{
A Critical Analysis of the Multi-Focus Images Fusion Using Discrete Wavelet Transform and Computer Vision
}

\author{
Gebeyehu Belay Gebremeskel \\ Bahir Dar University, Institute of Technology, Poly Main Campus, Agri Building 71, First Floor \\ ge,be09@yahoo.com
}

\begin{abstract}
This paper focused on the challenge of image fusion processing and lack of reliable image information and proposed multi-focus image fusion using discrete wavelet transforms and computer vision techniques for the fused image coefficient selection process. I made an in-depth analysis and improvement on the existing algorithms from the wavelet transform and the rules of multi-focus image fusion object features' extractions. The wavelet transform uses authentic localization properties, and computer vision provides efficient processing time and is a powerful method to analyze object focus in the highfrequency precision and steps. The process of image fusion using wavelet transformation is the wavelet basis function and wavelet decomposition level in iterative experiments to enhance fused image information. The rules of multi-focus image fusions are the wavelet transformation on the features of the high-frequency coefficients, which enhance the fusion image features reliability on the frequency domain and regional contrast of the object.
\end{abstract}

Keywords: multi-focus image; wavelet transformation; wavelet basis function; wavelet decomposing level; region contract, computer vision,

\section{Introduction}

Multi-focus image fusion is an effective technique to produce a single image by combining information from a set of source images to enhance object reality. It evolves various algorithms in a dynamic approach by extracting image similarities. Fusion techniques of an image can provide plentiful information by integrating the redundancy and complementary information that gain from the source images by forming a fused image (Vaishnavi and Jignyasa, 2019; Yong et al., 2014). The fusion image information content is reliable and valid as the image and efficient representation of the object features. The image features from different sources are an explicit description of the sequence of human to machine perception that an in-depth analysis of the image to present the objects focused on the same scene.

However, reliable information processing is challenging, which needs integrated multi-focus image fusion processing via (i) searching and selecting the relevant information of the fused image from the source images. (ii) Sequence and consistent image fusion processing lead to an efficient diagnosis. (iii) Fused image information taken from different levels of information representation. (iv) Image fusion in a single image focused on process and analysis for object region recognition (Harpreet and Rachna, 2015). This combined technique provides integrated visual information from different source images' edges and region focus for machine vision and object recognition.

Therefore, this paper proposes Discrete Wavelet transforms (DWT) and Computer Vision (CV) on multi-focus image fusion to in-depth analysis to enhance information quality (Fauhan and Aniati, 2019). Computer vision for wavelet image fusion is a technique to build an algorithm for a detailed image visual interface of extended image analysis (Suthakar et al., 2014; Zhang and Blum, 1999) to perform proper object localization (Sebastian, 2019). The image fusion technique supports multiscale and multiresolution transformations using frequency and spatial domain transform (Xiao, 2019; Radhika et al., 2017). The DWT-based image fusion demystifies data acquired from terraforming to correlate fused image features of the property of $\mathrm{CV}$ for image fusion using signal transform (Yihang et al., 2018). It evolves the coefficients of low-frequency and high-frequency sub-bands in different window-based fusion rules and processes (Velliangiri, 2019). The contribution of this paper is summarized as

- The DWT and CV based multi-focus image fusion technique presented and ensured the quality of the fusion results that suitable for human visual characteristics, and the critical analysis of fusion image, 
- It provides in-depth feature extraction from the source of images, and the gradient and the local visual contrast of the fused image through selecting the proper pixels as the object contexts,

- A discrete wavelet-based fused image is more suitable for human and machine perception using CV image processing and analysis to gain accurate image representations.

- It reduces the storage cost by the fused image instead of the multi-source image and improves the fusion performance.

The rest of the paper is organized as section 1 introduction in which briefly discusses the paper's rationale, research issues, and an overview of the algorithms and outcomes. Section 2 discussed and summarized the literature in detail. Section 3 is the methodology and technique of image fusion to enhance the quality of fusion information. Section 4 is the details of fusion algorithms and experiments. Sections $5 \& 6$ present and interpret the evaluation metrics analysis, which followed the conclusion.

\section{Related works}

DWT and CV-based multi-focus image fusion is the technique to process multiple images taken from the same scene. It focuses on different regions to extract image features to perform a critical analysis for information quality enhancement (Tao et al., 2013; Li, He et al., 2010). The fused image incorporates valuable features suitable for human/machine perception, image segmentation, feature selection, or object recognition. Various works include texture analysis, data compression, feature detection, etc. (Deepika and Mary, 2014; Yi et al., 2012) researched using wavelet transforms and computer vision. However, the integrity of DWT and CV gained more attention on the coefficient fusion to characterize the wavelet basis function and wavelet decomposition (Zhao et al., 2020).

As Devyani and Malviya (2015) studied, the wavelet basis functions are an experimental approach to determine the sub-image area ratio in wavelet decomposition and low/high-frequency. The low frequency contains the average intensity of the image. High frequency provides edge information (Agarwal et al., 2017). The rule of high or lowfrequency wavelet coefficients with the regional contrast indicates the transparent part of the picture by constructing the fused image (Zhan et al., 2017; Yushu et al., 2013). The concept and theory of DWT give a complete signal representation for CV involving fusion information (Andrey et al., 2018). CV-based image fusion analysis is a multi-resolution process to gain the fused image to enhance the DWT performance for image reality and spectral quality (Yong et al., 2014).

The DWT is a multi-sensor-based image fusion, which possesses symmetric, orthogonality, and short support (Nada et al., 2015). The image morphological wavelet decomposition scheme focuses on a simple arithmetic operation (De and Chanda, 2006) to enhance directional information by having the best signal-to-noise ratio, improved perception, etc. (Pajares and Cruz, 2004). The rules of multi-focus image fusion are used in different classifications, including signal, pixel, feature, and decision levels, using wavelet transforms. The process of selecting image features in a multi-resolution approach (Sarwar et al., 2021; Nada et al., 2018) is the wavelet transformation on the aspect of the high-frequency coefficients reflecting the best features of the regional contrast of the image (Vivek et al., 2019; Andrey et al., 2018). The fused image information is suitable to locate the object by having complete information (Li et al., 2019; Surabhi and Sameeksha, 2018). The integrity of DWT and CV is the time scale representation of the multi-focus fusion processing to enhance a fused image (Fauhan and Aniati, 2019; Suthakar et al., 2014). It passes different cutoff frequencies, scales, and levels by having detailed information (Yihang et al., 2018).

\section{III.The methodology: wavelet and computer vision integrity}

The integrated image fusion processing uses different algorithms to decompose the two-dimensional images into multi-resolution levels. A discrete set of wavelet scales' translations uses defined rules and information sources to create an improved CV (Natchammai and Hariharan, 2019). The integrity of wavelet and computer visions is efficient to consider all dimensions and focus in a single frame. The wavelet transforms a set of functions and signals analysis in both time and frequency domains simultaneously. The CV supports an image fusion interface 
development for the details of image features extraction to understand image contexts (Suthakara et al., 2014) for the same scene image to apply multi-focus image analysis (Chen et al., 2019). For in-depth visualization of an object, feature extraction is the fundamental step. At this level, the image classification process uses their similarities. The wavelet transfers and fusion algorithm using two registered images (I) of $\mathrm{P}_{1}\left(x_{1}, x_{2}\right)$ and $\mathrm{P}_{2}\left(x_{1}, x_{2}\right)$ represented as:

$$
I\left(x_{1}, x_{2}\right)=W^{-1}\left(\psi\left(W\left(p_{1}\left(x_{1}, x_{2}\right), p_{2}\left(x_{1}, x_{2}\right)\right)\right)\right)
$$

Where ' $\mathrm{W}$ ' is an operator of the wavelet transforms. $\mathrm{W}^{-1}$ is the inverse wavelet transforms, and ' $\psi$ ' is the fusion rule. The basic concept of wavelet-based image fusion analysis is the multiresolution decomposition of the source images, and the decomposed image coefficients combine using fusion rules (Meenu and Deepak, 2019; Nikita et al., 2017). The wavelet transform concentrates on representing the image in multi-scale and linear edges. For curved edges (nonlinear), apply a high accuracy of a curve localization, such as the curvelet transforms algorithm, which represents initially registered source images. The wavelet transform steps perform for each image to generate a set of wavelet coefficients. The inverse wavelet transforms for combined coefficient steps to gain the fused image (Tejashri and Alpana, 2015). It uses the analyses of a scalable window to cover the time-frequency to provide a convenient means for the non-stationary signals. The wavelet is constructed by multiplying wavelet functions $\psi(\mathrm{t})$ and scaling function $\varphi(t)$. The scaling functions are orthogonal to their discrete translations.

$$
\psi(t)=\sum_{k=-\infty}^{\infty} q_{k} \varphi(2 t-k) \text { and } \quad \varphi(t)=\sum_{k=-\infty}^{\infty} p_{k} \varphi(2 t-k)
$$

The various image fusion rules apply to select the coefficient of the focused region of the source images that provide clear fused image information (Kavitha S. and Thyagharajan K. K., (2017). The fusion image decomposition process describes the image size to halve the multi-resolution arrangements and establish the spatial directions using different techniques, including Haar, Coiflets, Daubechies, Symlets, etc. (Rajesh and Shivakumar, 2016). It is essential to avail effective metrics to measure the clarity of source images (Maruthi and Lakshm, 2017). The DWTbased fusion scheme processing is a sequential step (figure 1) to decompose the source images into sub-images and then combine them using a fusion rule to gain a fusion image. Assess fused image processing using quality indicators that construct concepts of a reference image to compare with the experimental results.



Figure 1 the proposed DWT based multi-focus image fusion flow diagram 
The DWT technique in figure 1 showed the process of image decomposition and a multi-stage transformation (Wu et al., 2012) into low and high sub-bands, which is the Low-Low band of the current decomposition stage. The Nlevel image decomposition process in $3 \mathrm{~N}+1$ different frequency bands, including $3 \mathrm{~N}$ high-frequency bands and one Low-Low frequency band. For the empirical analysis, I use the mean value, standard variation, information entropy, correlation coefficient, (root) mean squared error (MSE), peak signal to noise ratio (PSNR), etc. (Blasch et al., 2008) to evaluate the performance of image fusion results. The proposed approach addresses:

- Search the optimal wavelet basis function and decomposition level of source images.

- Perform DWT in two dimensions on each image fused to get coefficient vectors.

- The coefficient vectors of high frequency and low frequency are separately computed with different operators then integrated as a new image's wavelet transformation coefficient vector.

- Develop fusion rules and process the invert decomposed image. The reverse DWT executes on the fused coefficient vector to achieve a new image fusion,

- Evaluate the performance of the image fusion algorithm by measuring the quality of the fusion image.

\subsection{Multi-focus image fusion methods}

Image fusion performs in a multi-focus, multi-view, multi-modal, and multi-temporal way. The multi-focus image fusion algorithm is to identify focused regions using fusion rules. It is a technique applying the evaluating metrics for the blurred image and selecting information from the sharper image by considering the wavelet coefficients (Yong et al., 2014). It processes as the category of frequency and spatial domain methods, which involves an image passing multiple levels of resolutions followed by various manipulations of the image transformation (Qiang et al., 2019). The fusion method based on the transforms domain gains better fusion results (Meenu and Rajiv, 2017; Roosta et al., 2015) by selecting appropriate pixels or regions within the source image to construct the fused image, as shown in figure. 2 .

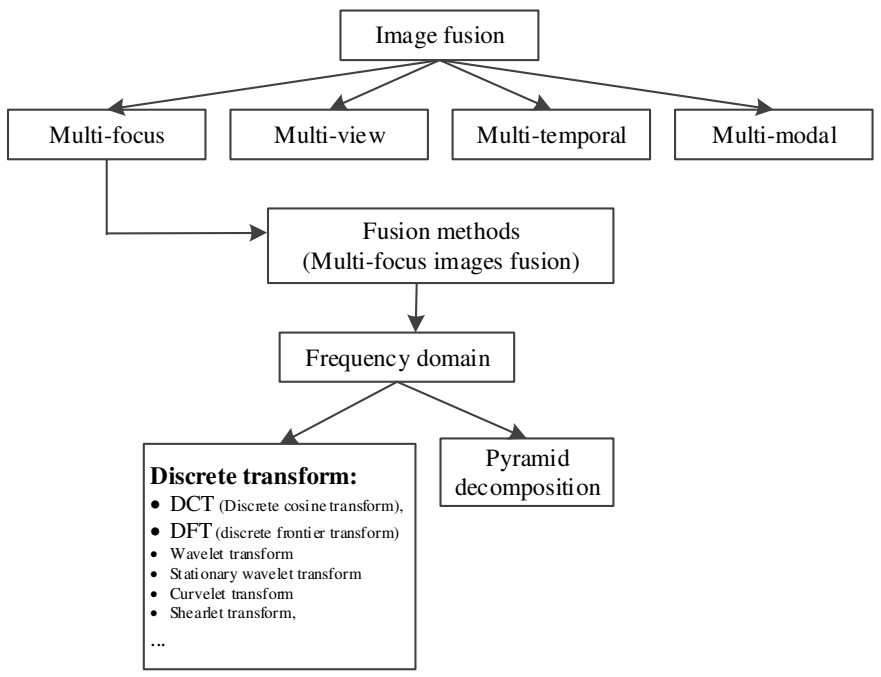

Figure 2: Image fusion and the methods used in multi-focus image fusion

\subsection{Frequency domain image fusion}

Frequency-domain methods decompose the input images into multi-scale coefficients using various algorithms, including Discrete Cosine Transform (DCT) or Discrete Fourier transforms (DFT). The fusion rules employ to select these coefficients and synthesize the inverse transform of fused images (Maria et al., 2020; Dhirendra and Bhakti, 2015). The coefficients at each frequency determine inverse transforms to construct the fusion image.

The discrete wavelet-based fusion is applied to split the input images into sub-parts based on band coefficients. The fusion has then fused these coefficients using the technique of possible selection. The fusion image is the result of DWT Inverse on the combined coefficients. The wavelet mapping depicted different types of wavelet transforms in 
the fusion process. As shown in figure 3, the frequency domain-based image fusion process reveals the various forms of wavelet transforms.

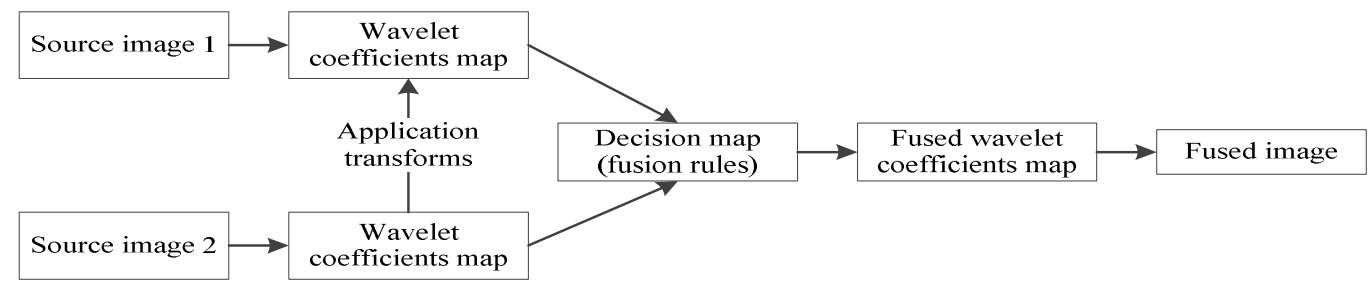

Figure 3: Frequency-domain image fusion process

\subsubsection{Wavelet transform based fusion}

The wavelet transform is an image decomposition and reconstruction technique, which evolves fusion rules to measure the performance of the fused image. It uses multi-criteria to select the coefficients of the focused region from the source images. The wavelet transform is a multi-focus image fusions technique. It applies to gain a single fused image using various schemes of morphological and technical algorithms. It passes integrated features, pixels, and decision level fusion to extract local features in detail sub-bands (Alberto et al., 2010). In nonlinear wavelet constructs, morphological wavelet and multi-wavelet transform with Haar function, bi-orthogonal wavelet, and redundant wavelet transform are efficient approaches (Ma et al., 2020).

Wavelet transforms in multi-focus image fusion are for multi-resolution analysis. For nonlinear wavelets, machine vision applies to enhance a fused image similarity. The nonlinear process is the process to improve the decomposed signal image within the context of the exact object features, which uses sources image morphological operators and a lifting scheme. It involves division operation for floating-point or integer arithmetic. The techniques include DWT, Complex Wavelet transforms (CWT), Curvelet transforms (CT), Fast Discrete Curvelet transforms (FDCT), Shearlet transforms (ST), etc., for the fusion of multi-focus images.

\subsubsection{Discrete wavelet transforms fusion}

DWT is a wavelet-based transform to record the election results based on a maximum selection rule. It provides a compact representation of the signal's frequency component by achieving better frequency information and realtime resolution. DWT method employs in the human perceptual domain based on the frequency response. The fusion of image contrast and sensitivity of the human prospect focused on image decomposition into frequency subbands at different scales by splitting into high and low frequency (Tian et al., 2018). The source images initially transform into multi-resolution representations of the wavelet coefficients are gained using a weighted average on the local activity levels in each of the images' sub-bands.

DWT applies in many fields associated with a given pixel, which uses as the activity measure. It applies to every image block assumption that images focus on Buller areas than individual pixels. In this way, a high activity value indicates a dominant feature in the local area. The image fusion process uses fusion rules to obtain a new composite multi-resolution representation (Ting et al., 2019). A conventional fusion scheme is selected the absolute wavelet coefficient at each location within the source image. In DWT, the image signals generate a non-redundant image features representation for improved spatial and spectral localization fusing image formation. It is the best representation in the Gaussian and Laplacian pyramids (Kulvir and Neeraj, 2016).

\subsubsection{Discrete cosine transforms}

In real-time, the discrete cosine transforms (DCT) based image fusion is suitable to save processing time. And also, it is an efficient method for image coding. The DCT is an efficient tool in the Joint Photographic Experts Group (JPEG) format. 
The DCT is a frequency-domain algorithm to determine the coefficients of each transform and perform the inverse transform to construct the fused image. It is a process to avoid discontinuity transition.

\subsubsection{Curvelet transforms}

The Curvelet transforms (CT) is a multi-scale transform. It is suited for objects from discontinuities across curves. Curvelet transform handles curve discontinuities well as they design to handle curves using only a small number of coefficients.

Curvelet is essential to consider long edges for the wavelet transform process. It employs edges as the fundamental element and possesses maturity, anisotropy, high directional sensitivity to provide more image information to adapt well image characteristics. CT can represent the edge of the image and smoothness area in the same precision of inverse transforms. It is essential to optimize the integrity of the image fusion process and computer vision.

\subsubsection{Shearlet transforms (ST)}

The shearlet is an emerging multi-scale geometric analysis technique to enhance the visual quality of the fused image (Ahmed et al., 2019). ST has a mathematical structure similar to wavelets for multiresolution analysis. The shearlet is in multiple directions and dynamic image processing applications, including image denoising, sparse image representation, and edge detection (Sankaran and Nagarajan, 2019; Li et al., 2018). It works in the highfrequency coefficients based on regional energy, variance, and the absolute value of detailed information in any scale and direction.

The Non-Sampled Shearlet transforms (NSST) is the shift-invariant version of ST that captures a 2-D geometrical structure much more effectively than the traditional Multi-Scale transforms (MST). The fundamental aspect of ST is to eliminate the down-samplers and up-samplers, whereas NSST is to gain more information for fusion (Lu et al., 2017; Nianyi et al., 2015) to select the fusion coefficient.

\subsubsection{Stationary Wavelet transforms}

Stationary Wavelet transforms (SWT) are the same as DWT, which performs the down-sampling delete and translation variant with an equal size of the low and high frequencies sub-bands. SWT-based multi-focus image fusion technique is an extended Spatial Frequency Measurement (SFM) and wavelet filter for orthogonal and biorthogonal wavelets methods (Sarwar et al., 2021; Toet, 1989). A multi-focus color image fusion method is implemented to perform Intensity-Hue-Saturation (IHS) transform on multi-focus color images to get intensity components and wavelet coefficients by taking SWT.

An SWT-based multi-focus image fusion method is a multi-scale product in the redundancy of $2 \mathrm{~N}$ disintegration into N levels (Radhika et al., 2017). SWT is a surplus system for every decomposed image group of coefficients similar features amount on the source images. SWT with DWT is a method to translate the invariance of the wavelet coefficients. The redundancy makes it possible to recognize significant features in a signal of the object and the noise detections.

\section{Image fusion algorithms}

The multi-focus fusion algorithm of an image is the process to preserve the salient features of the source image (edges, textures, etc.), avoiding non-consistent information that affects the quality of the fused image and the subsequent processing (Qiang et al., 2020). It needs to robustness to the registration error and noise in the source images. The algorithms focused on image region and localizations of pixels to accept the implicit assumption to gain local changes in the intensity of consistent human visual perception (Meenu and Deepak, 2019). It addresses using the known reference and the variant of the images, entropy, similarity, and other measures (Li et al., 2018; Manu and Philomina, 2012). 
The regions of the images in focus give rise to more generous magnitude coefficients, in which a maximum scheme produces the merged coefficient map. The mapping fused image is transformed into the combined coefficient map using the inverse intricate wavelet transform. The wavelet coefficient images gained the orientated nature of the complex wavelet sub-bands. It performs a superior fused image from the source images by extracting specific features to classify as spatial and frequency-domain fusion.

\subsection{Haar wavelet transforms}

The fundamental process of the Haar wavelet transform is to possess many attractive features, including orthogonal, compact, and infinite supports. It uses the frequency domain, symmetric in scaling function and anti-symmetric in wavelet function. The Haar wavelet transforms characteristics to the fusion of an image that performs accurate, robust, and memory-efficient tasks. Haar is an integral-based image technique for multi-focus applications to various fields such as object recognition, feature extraction in image processing, and object localization of military surveillance and aeronautical observation images.

Haar wavelet is a sequence of rescaled equal-sized shapes to form a wavelet family for wavelet and Fourier's analyses. It allows a target function over an interval to represent an orthonormal function basis, which considers a sequence and recognizes the first known wavelet basis of the wavelet and scaling function. The Haar wavelet function $\psi(t)$ described as:

$$
\psi(t)=\left\{\begin{array}{cc}
1 & 0 \leq t<1 / 2, \\
-1 & 1 / 2 \leq t<1, \\
0 & \text { otherwise }
\end{array} \text { and Haar scaling function } \varphi(t) \text { can be described as } \varphi(t)=\left\{\begin{array}{cc}
1 & 0 \leq t<1, \\
0 & \text { Otherwise }
\end{array}\right.\right.
$$

\subsection{Daubechies wavelet transforms}

Daubechies wavelet is an orthogonal wavelet to perform the maximum number of vanishing moments, which supports image coding and testing. It employs continuous and discrete transforms to enhance the scaling function generates the orthogonal multiresolution.

\subsection{Coiflets wavelet transform}

Coiflets wavelets are discrete and characterized as the scaling function with many vanishing moments. It has $\frac{N}{3}$ number of vanishing moments in wavelet function and $\frac{N}{3}$-1vanishing moments in scaling function that normalized by $\frac{1}{\sqrt{2}}$.

\subsection{Symlets wavelet transforms}

Symlets wavelet supports compacted scaling and wavelet functions known in orthogonal wavelets. It is an efficient approach to handling low regularity source images.

\subsection{Biorthogonal and Reverse Biorthogonal}

The biorthogonal method is invertible and competent to construct a symmetric wavelet function. It supports a dynamic wavelet and scaling function. The user applications and reconstruct needs the wavelets to perform in the image fusion process. Once the decomposition did the fusion rules combine the detail coefficients into fusion coefficients, the IDWT (Inverse Discrete Wavelet Transform) converts into the fused image of a choice of wavelets to the user.

\subsection{Mallat Algorithm}

Mallat is a quicker algorithm based on multiple resolution analyses of wavelet transformation (Mallat, 1989; Aggarwal, 1993) and provides a powerful tool during the image fusion process. It is an orthogonal decomposition of the image onto a wavelet basis to avoid the redundancy of information in the pyramid at each level of resolution. 
The two fundamental processes of the Mallat algorithm are decomposition and construction, which are discussed in detail as follows.

\subsubsection{Decomposition}

The source image is filtered by a low pass (L) and high pass $(\mathrm{H})$ in the horizontal direction and down-sampled by a factor to get the coefficients matrices $\operatorname{Im}(\mathrm{L})$ and $\operatorname{Im}(\mathrm{H})$. Same filters with down-sampling applied to coefficient (C) matrices in a vertical direction to obtain sub-bands, $\operatorname{Im}(\mathrm{LL}), \operatorname{Im}(\mathrm{LH}), \operatorname{Im}(\mathrm{HL})$, and $\operatorname{Im}(\mathrm{HH})$ are images with a low-low, low-high, high-low, and high-high frequency, respectively (Li et al., 2019; Tian et al., 2018; Agarwal et al., 2017). The wavelet decomposition formulated in the eq. (4-7).

$$
\begin{aligned}
& C_{j+1}=H_{r} G_{c} C_{j} \\
& D_{j+1}^{1}=H_{r} G_{c} C_{j} \\
& D_{j+1}^{2}=G_{r} H_{c} C_{j} \\
& D_{j+1}^{3}=G_{r} G_{c} C_{j}
\end{aligned}
$$

Where $\mathrm{j}=0,1,2 \ldots \mathrm{j}-1$ is for decomposition level. $H$ and $G$ represent the low-pass and high-pass filtering; $H_{r}$ and $G_{r}$ are an expression of filtering operated on the row; $H_{c}$ and $G_{c}$ are the expressions operated on the column. Wavelet decomposition is implemented on the low-frequency segmentation of the upper level decomposed, and then the pyramid structure of wavelet transformation forming. And D is the fused image discrete details in horizontal, vertical, and diagonal expression (Cai et al., 2020).

\subsubsection{Reconstruction}

The technique of Inverse DWT is to reconstruct a 2D image from sub-bands $\operatorname{Im}(\mathrm{LL}), \operatorname{Im}(\mathrm{LH}), \operatorname{Im}(\mathrm{HL})$, and $\operatorname{Im}(\mathrm{HH})$, as depicted in figure 4, which involves column upsampling for low and high pass filters for each subband image. The same filters apply with row-up sampling to get the fused image. The algorithm of reconstruction is modeled by the eq.8, where $H^{*}, G^{*}$ represents the reverse transformation compared with $\mathrm{H}$ and $\mathrm{G}$. Image reconstruction equation is presented as:

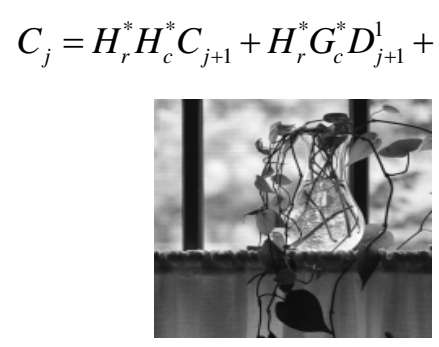

(a)The image of $\mathrm{Cj}$

$$
+G_{r}^{*} H_{c}^{*} D_{j+1}^{2}+G_{r}^{*} G_{c}^{*} D_{j+1}^{3}
$$



(b)Image after 2 levels of decomposition

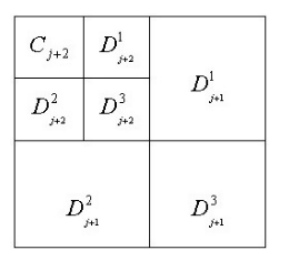

(c)The coefficient distribution

Figure 4 Distribution of image wavelet coefficient

\section{Performance measuring techniques}

The multi-focus fusion image performance measuring using reference and without reference images. It applies in various fusion methods, including entropy, RMSE, PSNR, image quality index, etc.

\subsection{Entropy}

Entropy $(\mathrm{H})$ is the technique to measure information content present in an image and sensitivity to noise and unwanted rapid changes (Zheng and Wang, 2018). If the entropy is higher in the fused image, it carries quality 
information to the source image. The higher the value of entropy to the whole range and the similar frequency, the information content of the fused image is higher than the source images. And its mathematical formulation is:

$$
H=\sum_{g=0}^{L-1} p(g) \log _{2} p(g)
$$

Where $\mathrm{p}(\mathrm{g})$ is the source image, and $\mathrm{p}$ is the distribution probability of the gray (intensity) value of an image. ' $\mathrm{L}$ ' is the total number of the gray (blurred) level.

\subsection{Root Means Square Error (RMSE)}

The RMSE evaluates the deviation present in the fused image compared to the source image. It is a reference-based assessment measuring technique of fused and source image information. RMSE value must be as low as possible for better image quality. It computes as the root means square error between a reference image ' $R$ ' and a fused image, 'F' as:

$$
R M S E=\sqrt{\sum \sum 1 / M N(R(m, n)-F(m, n))^{2}}
$$

$\mathrm{R}(\mathrm{m}, \mathrm{n})$ and $\mathrm{F}(\mathrm{m}, \mathrm{n})$ are the reference and fused image points, respectively, and $\mathrm{M}$ and $\mathrm{N}$ are image dimensions. The smaller the value of the RMSE, is the better the performance of the fusion algorithm.

\subsection{Peak Signal to Noise Ratio (PSNR)}

PSNR measures the maximum value of an image ratio and the magnitude of the noise present in the image. It improves the quality of the fused image by the maximum possible power of a signal, and the power of corrupting noise affects the fidelity of its representation. It is the technique to construct the quality of the fused image or the similarity between two images (Xueping et al., 2019). The range of this metric is -1 and +1 , and the best value is +1 to achieve if and only if the reference and fused images are similar. The higher PSNR value gives the better-fused image quality, and its mathematical formulation is:

$$
P S N R=10 \times \log \left(\frac{f \max ^{2}}{R M S E^{2}}\right)
$$

The symbol $f$ max represents the maximum grayscale value of the pixels in the fused image. The higher the value of the PSNR, is the better the performance of the fusion algorithm.

\subsection{Clarity (Quality Index)}

The quality index measures the salient information obtained in the reference image that transforms into the fused image. It is a distortion of a combined loss of correlation, luminance, and contrast. It formulated as:

$$
Q I=\frac{\left(4 \times \sigma_{I F}\right)\left(\mu_{I}+\sigma_{F}\right)}{\left(\mu_{I}^{2}+\mu_{F}^{2}\right)\left(\sigma_{I}^{2}+\sigma_{F}^{2}\right)}
$$

Where $\sigma I F$ is the covariance of $I F, \mu F$ is the average intensities of image $F$, and $\mu I$ is the average intensities of the image $I, \sigma F 2$ denotes the variance of image $F$ and $\sigma I 2$ is the variance of the image $I$. The QI value varies from - 1 to 1 , and the value close to +1 indicates the similarity of the two images.

\subsection{Fusion-based standard deviation (SD)}

The standard deviation is a technique to reflect the image clarity and contrast information. The standard deviation uses to calculate the proportion of the gray value that spreads in an image, and the smaller the SD, is the image is free of noise. However, the smaller the image contrast is, the more affected by noise: 


$$
S D=\sqrt{\frac{1}{m \times n} \sum_{x=1}^{m} \sum_{y=1}^{n}(I(x, y)-\bar{I})^{2}}
$$

\subsection{Fusion-based mean value}

The mean value technique applies to large-scale data set of fusion images to represent a value that describes the center or average rate, which measures the set of data contributions of the same amount by each $n$ contributor. The statistical formula is:

$$
\bar{x}=\frac{1}{n} \sum_{i=1}^{n} x_{i}
$$

The mean value is computed on input image low-frequency components within a square window, and higher values of the mean are used to select the fusion coefficients of the low-frequency components.

\subsection{Correlation entropy}

Correlation entropy measures feature information between the standard reference and fused images. The volume of feature information of fused image-standard reveals the correlation entropy, which takes into account luminance structure, correlation, and contrast. Correlation entropy (CE) is defined as the luminance of the quality, and the contrast similarities, respectively:

$$
C E=\frac{\left(\sum_{m=1}^{M} \sum_{n=1}^{N}(R(m, n)-\bar{R}(m, n))(F(m, n)-\bar{F}(m, n))\right)}{\sqrt{\left(\sum_{m=1}^{M} \sum_{n=1}^{N}(R(m, n)-\bar{R}(m, n))^{2} \times \sum_{m=1}^{M} \sum_{n=1}^{N}(F(m, n)-\bar{F}(m, n))^{2}\right)}}
$$

$\bar{R}(m, n)$ and $\bar{F}(m, n)$ are the pixel gray average value of the standard reference image of $\mathrm{R}$ and fused image $\mathrm{F}$, respectively. The measures show the degree of matching between the fused and the standard of the reference image, and the larger the value is, the better the fusion effect.

\subsection{Spatial frequency (SF)}

The spatial frequency (SF) measuring technique is the overall contexts and clarity level of the fused image. If a block of image $F$ of size ' $M$ ' by ' $N$ ' has a gray value of $F(m, n)$ at position $(m, n)$, then the value of SF is defined as eq.16-18. The SF value represents the better fusion result.

$$
S F=\sqrt{R F^{2}+C F^{2}}
$$

Subject to: RF (row frequency)

$$
R F=\sqrt{\frac{1}{(M-1) N} \sum_{m=0}^{M-1} \sum_{n=0}^{N-2}(F(m, n-1) F(m, n))^{2}}
$$

CF (Column Frequency)

$$
C F=\sqrt{\frac{1}{(M-1) N} \sum_{m=0}^{M-2} \sum_{n=0}^{N-1}(F(m+1, n) F(m, n))^{2}}
$$




\subsection{Fusion mutual information (FMI)}

FMI is a fusion measuring factor of the degree of the correlative information content of the source and fused images. The larger the value of FMI implies better quality, which is computed from two images of $x_{F}$ and $x_{R}$, it formulated as:

$$
F M I=I\left(x_{A} ; x_{F}\right)+I\left(x_{B} ; x_{F}\right)
$$

Subject to

$$
\begin{gathered}
F M I_{A F}=\sum_{i=1}^{M} \sum_{j=1}^{N} p A, F(i, j) \log _{2} \frac{p A, F(i, j)}{p A(i) p F(j)} \\
F M I_{B F}=\sum_{i=1}^{M} \sum_{j=1}^{N} p B, F(i, j) \log _{2} \frac{p B, F(i, j)}{p B(i) p F(j)}
\end{gathered}
$$

The FMIAF and FMIBF are techniques to measure the quantity of mutual information between source images A \& $\mathrm{B}$ and fused image $\mathrm{F}$ accordingly. The FMIAB measures the overall mutual information between the source image and the fused-image quality. $\mathrm{P}$ is the normalized gray level distribution probabilities of $x_{R}$ and $x_{F}$, respectively.

\subsection{Cross entropy $\left(\mathrm{H}_{\mathrm{CE}}\right)$}

Cross entropy is also a measure of the differences between the probability distribution of information contents of sources and fused images. It is calculated from source images $\mathrm{p}(\mathrm{x})$ and fused image $\mathrm{q}(\mathrm{x})$ is formulated as:

$$
H_{C E}(q, p)=\sum_{\forall x} q(x) \log \frac{q(x)}{p(x)}
$$

\subsection{Joint entropy $\left(\mathrm{H}_{\mathrm{JE}}\right)$}

The joint entropy is the amount of information needed on average to specify the value of two discrete random variables by observing the two images at the time. Let ' $\mathrm{X}$ ' and ' $\mathrm{Y}$ ' be discrete random variables, and $\mathrm{f}(\mathrm{x}, \mathrm{y})$ is the value of their joint probability distribution at $(\mathrm{x}, \mathrm{y})$. And the joint entropy of ' $\mathrm{X}$ ' and ' $\mathrm{y}$ ' is:

$$
H_{J E}(X, Y)=-\sum_{x \in X} \sum_{y \in Y} f(x, y) \log f(x, y)
$$

\section{Experiment and analysis of wavelet basis and decomposition level}

Nowadays, the wavelet transform approach applies in a wide area, including fusing data, and it is a hot topic in research. Wavelet transforms implements to decompose and explore the effects of different wavelet coefficients for the fusion images in spectral and spatial features. This part will analyze how they affect the result of fusion regarded as a guide for the later fusion algorithms. In this paper, the source image scenes are registered and prepared for DWT-based image fusion.

\subsection{Selection on a wavelet basis on image fusion}

The multi-focus image fusion based on the wavelet transformation depends on the wavelet transform coefficients in various dimensions. The distribution of wavelet transforms coefficients is highly influenced by a wavelet basis function. Selecting a proper wavelet basis is the pivotal factor of the quality of the fusion image, and the properties in the wavelet family summarize in Table 1. 
Table 1 Performance indicators of wavelet basis

\begin{tabular}{|c|c|c|c|c|c|}
\hline Performance indicators & Haar & $\begin{array}{l}\text { Daubechies } \\
(\mathbf{d b})\end{array}$ & BiorSplines (bior) & Coiffets (coief) & Symlets (Sym) \\
\hline Name & Haar & $\mathrm{dbN}$ & Burner.Nd & coifN & $\operatorname{symN}$ \\
\hline Orthogonal & $\mathrm{N}$ & $\mathrm{N}$ & $\mathrm{N}$ & $\mathrm{Y}$ & $\mathrm{Y}$ \\
\hline Bi-orthogonal & $\mathrm{Y}$ & $\mathrm{Y}$ & $\mathrm{Y}$ & $\mathrm{Y}$ & Y \\
\hline $\begin{array}{l}\text { Compact support } \\
\text { length }\end{array}$ & 1 & $2 \mathrm{~N}-1$ & $\begin{array}{l}\text { DEC: } 2 \mathrm{Nd}+1 \\
\text { REC: } 2 \mathrm{Nr}+1\end{array}$ & $6 \mathrm{~N}-1$ & $2 \mathrm{~N}-1$ \\
\hline Filter length & 2 & $2 \mathrm{~N}$ & $\max (2 \mathrm{Nd}, 2 \mathrm{Nr})+2$ & $6 \mathrm{~N}$ & $2 \mathrm{~N}$ \\
\hline Symmetry & Symmetry & Nonsymmetric & Symmetry & $\begin{array}{l}\text { Similar } \\
\text { Symmetry }\end{array}$ & $\begin{array}{l}\text { Similar } \\
\text { Symmetry }\end{array}$ \\
\hline $\begin{array}{l}\text { Vanishing moment of a Scaling } \\
\text { function }\end{array}$ & none & none & none & $2 \mathrm{~N}-1$ & none \\
\hline $\begin{array}{l}\text { Vanishing moment of Wavelet } \\
\text { function }\end{array}$ & 1 & $\mathrm{~N}$ & $\mathrm{Nr}-1$ & $2 \mathrm{~N}$ & $2 \mathrm{~N}$ \\
\hline
\end{tabular}

\section{Experiment 1}

This experiment is focused on how the wavelet basis affects the quality of fusion images by undertaking the iterative process on image data, as shown in figure 5, with different wavelet basis functions. The fusion algorithm applied in transforming wavelet coefficients is a pixel average. The quantitative measurements of the fusion images have presented in table 2 .

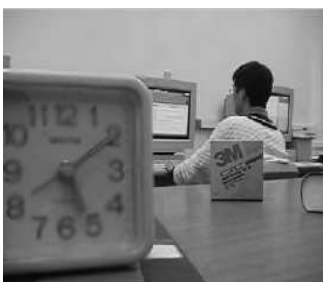

a) Image focused on the person

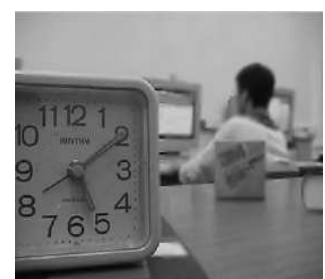

(b) Image focused on the clock

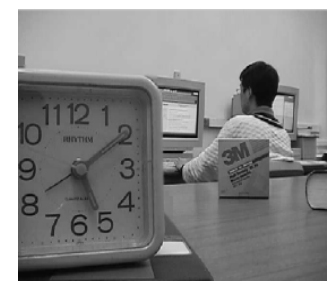

(c) the reference image

Figure 5 Multi-focus images of clock and person

Table 2 the result of pixel-based image fusion with different wavelets

\begin{tabular}{cccccccc}
\hline $\begin{array}{c}\text { Wavelet } \\
\text { basis }\end{array}$ & Mean value & $\begin{array}{c}\text { Standard } \\
\text { variation }\end{array}$ & Entropy & Clarity & MSE & PSNR & $\begin{array}{c}\text { correlation } \\
\text { Entropy }\end{array}$ \\
\hline Haar & 123.2311 & 45.8482 & 6.9841 & 4.8854 & 5.4948 & 32.8870 & 0.3165 \\
Db2 & 123.2038 & 45.8659 & 6.9936 & 4.8863 & 5.4881 & 32.8976 & 0.3926 \\
Db4 & 123.1981 & 45.8605 & 6.9958 & 4.8837 & 5.4902 & 32.8943 & 0.3866 \\
Db6 & 123.1735 & 45.8874 & 6.9985 & 4.8858 & 5.4811 & 32.9086 & 0.3938 \\
Db8 & 123.1816 & 45.8930 & 7.0061 & 4.8850 & 5.4821 & 32.9070 & 0.3684 \\
Sym2 & 123.2038 & 45.8659 & 6.9936 & 4.8863 & 5.4881 & 32.8976 & 0.3946 \\
Sym4 & 123.1740 & 45.8794 & 6.9923 & 4.8824 & 5.4909 & 32.8931 & 0.3996 \\
Sym6 & 123.1855 & 45.8876 & 6.9925 & 4.8833 & 5.4877 & 32.8981 & 0.3941 \\
Sym8 & 123.1726 & 45.8780 & 6.9929 & 4.8832 & 5.4822 & 32.9068 & 0.3941 \\
\hline
\end{tabular}




\begin{tabular}{llllllll}
\hline Coif2 & 123.1823 & 45.8697 & 6.9896 & 4.8811 & 5.4889 & 32.8963 & 0.4029 \\
Coif4 & 123.1753 & 45.8709 & 6.9909 & 4.8824 & 5.4831 & 32.9054 & 0.3989 \\
Coif5 & 123.1704 & 45.8703 & 6.9969 & 4.8818 & 5.4842 & 32.9037 & 0.3910 \\
Bior2 & 123.6249 & 45.8872 & 6.9957 & 4.8900 & 5.4364 & 32.9060 & 0.3997 \\
Boir4 & 123.2194 & 45.8845 & 6.9983 & 4.8860 & 5.4047 & 32.9713 & 0.4017 \\
Bior5 & 123.0506 & 45.8873 & 6.9836 & 4.8779 & 5.4788 & 32.9123 & 0.4183 \\
Bior6 & 123.2052 & 45.8631 & 6.9942 & 4.8821 & 5.4847 & 32.9030 & 0.3931 \\
\hline
\end{tabular}

From the quantitative measurements of the fusion images, the affections of the wavelet basis function on image fusion are the reality of the fused image. The metrics techniques are the quality of information that gain from the fusion image, which implements for various applications, including monitoring image fusion, optimization algorithms, and data settings of image fusion to define the quality measures in the context of source images.

\subsection{Optical numbers of wavelet decomposition level}

Wavelet decomposition is a process of image segmentation for image spot localization using time and frequency domains in detailed object focus. It provides a powerful method for multi-focus image fusion (David, 1995; Zhang and Blum, 1999). The number of decompositions was determined using the area ratio and MSE.

Definition 1: the area ratio (r) of sub-images defined as:

$$
r=\frac{S_{k}}{S_{k-1}}
$$

Where $\mathrm{k}$ is the number of wavelet decomposition level; $S_{k}$ is the sub-images are on that level; $S_{k-1}$ is the sub-images area of the level k-1;

Definition 2: mean-square error (MSE) is used to measure the deviations between the source and the fused image. MSE is an error magnitude measure derived from the squared error and the total input pixels in the image region. The smaller the error metric value is, the better the fused image quality. Supposing that low bands on the k level of wavelet decomposition presented as $\mathrm{Mk}$ and $\mathrm{Nk}$ in two source images, and it defined as:

$$
M S E_{k}=\sqrt{\frac{\sum_{i=1}^{m} \sum_{j=1}^{n}\left(M_{k}(m, n)-N_{k}(m, n)\right)^{2}}{m \times n}}
$$

Where ' $m$ ' and ' $n$ ' are the dimensions in the low band, which is the algorithm for an optimal number of wavelet transformation levels.

Algorithm 1: The optimal number of wavelet transformation level

1. Compute the smallest number ' $m$ ' of wavelet decomposition level whose sub-images area is larger than 0.5 and the biggest number ' $n$ ' of wavelet decomposition level whose sub-images region is smaller than 0.5 satisfying $m=n+1$

2. Compute the $\mathrm{MSE}_{m}, \mathrm{MSE}_{n}$ on the level of ' $m$ ' and ' $n$ '

3. The optimal number of decomposition level $\mathrm{K}$ equals to ' $m$ ' if $\mathrm{MSE}_{m}<\mathrm{MSE}_{n}$ else equals ' $n$ '

4. Quit.

\section{Experiment 2}

In this experiment, two source images (figure 6) are focused on different regions. And one reference image is 
implemented using the Matlab wavelet toolbox based on the db6 wavelet basis on the two source images whose size is $385 \times 386$. The sub-image ratio is 0.5291 , while the MSE is 8.4862 in an optimal wavelet composition level is five. Table. 3 provides a quality evaluation of the populate image fusion using the wavelet decomposition level variable from one to ten. The result for spatial frequency reaches its maximum when the number of the wavelet decomposition levels is five (Yogita and Andurkar, 2013). The entropy value increases with a few changes of wavelet decomposition with a similar value of MSE. The computational complexity increases and the fusion images represent in (d) and (f) with the wavelet composition level of $1,5,10$.

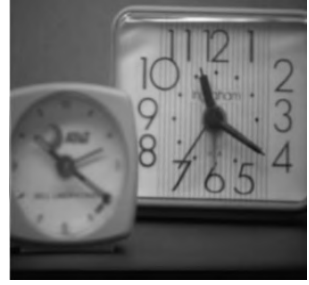

(a) The image focused on Left

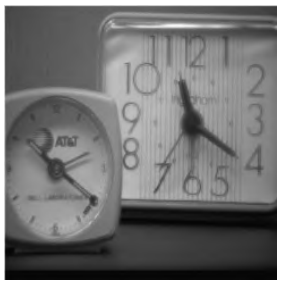

(d)The resulting image of $n=1$



(b) The image focused on the right

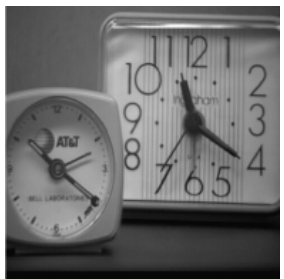

(c) The reference image

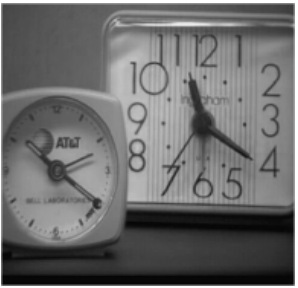

(e) The resulting image of $n=5$



(f) The resulting image of $n=10$

Figure 6. The original reference image, Gaussian blurred images, and results of fusion with different levels of wavelet decomposition

Table 3: The comparison among fusion effect of the objective with different decomposition levels and wavelet energy

\begin{tabular}{cccccc}
\hline $\begin{array}{c}\text { Fusion image based on } \\
\text { wavelet energy }\end{array}$ & Entropy & $\begin{array}{c}\text { Spatial } \\
\text { Frequency }\end{array}$ & RMSE & $\begin{array}{c}\text { Mutual } \\
\text { Information }\end{array}$ & Cross-Entropy \\
\hline N=1 & 7.4015 & 10.5203 & 8.4928 & 0.5963 & $\mathbf{2 2 . 1 8 5 6}$ \\
N=2 & 7.4123 & 12.3801 & 6.1156 & 0.6207 & $\mathbf{2 2 . 2 1 9 9}$ \\
N=3 & 7.4143 & 13.1678 & 3.0665 & 0.6559 & $\mathbf{2 2 . 2 3 9 0}$ \\
N=4 & 7.4078 & 13.2488 & 1.8110 & 0.6631 & $\mathbf{2 2 . 2 1 6 5}$ \\
N=5 & 7.4050 & 13.2508 & 1.6466 & 0.6650 & $\mathbf{2 2 . 0 7 7 7}$ \\
N=6 & 7.4051 & 13.2511 & 1.6269 & 0.6649 & $\mathbf{2 2 . 2 0 7 6}$ \\
N=7 & 7.4053 & 13.2508 & 1.6179 & 0.6651 & $\mathbf{2 2 . 2 0 8 5}$ \\
N=8 & 7.4044 & 13.2520 & 1.5826 & 0.6649 & $\mathbf{2 2 . 2 0 5 1}$ \\
N=9 & 7.4043 & 13.2519 & 1.5802 & 0.6649 & $\mathbf{2 2 . 2 0 4 8}$ \\
N=10 & 7.4044 & 13.2518 & 1.5826 & 0.6649 & $\mathbf{2 2 . 2 0 5 4}$ \\
\hline
\end{tabular}

Table 3 shows the better-referring information to the levels of wavelet decomposition in multifocal image fusion that is necessary to find the wavelet transformation.

\subsection{The improved algorithm for wavelet-based image fusion}

In the wavelet theory of Mallat, an image transformation performs using a discrete wavelet in two dimensions. The Mallat gains frequency bands in four directions (level, perpendicular and diagonal) (Xueping et al., 2019). The improved image fusion is based on the DWT algorithm, and each source image is transformed by a discrete wavelet 
segmented into sub-images within low and high frequencies. The characteristic in low and high frequencies operator based on the region contrast introduced to improve the quality of fusion images.

\subsubsection{The improved algorithm for coefficients in low frequency}

The information in low frequency, which affects the energy of the fusion image and plays a decisive role in light of the fusion image, is similar to the father image. So it's not suitable to operate the coefficient in low frequency in the region, which will cause the light decrease to mean an infusion image. Considering the importance of the pixel, I improve the traditional simple average method by constructing a weight for each source coefficient, and then the fusion operation is defined as eq. 26.

$$
F_{A}(x, y)=\alpha A_{A}(x, y)+(1-\alpha) B_{A}(x, y)
$$

Where $F_{A}(x, y)$ is the coefficient of the fusion image, while $A_{A}(x, y)$ and $B_{A}(x, y)$ are the coefficients in low frequency from source image $\mathrm{A}$ and $\mathrm{B}$ with the same position $(\mathrm{x}, \mathrm{y})$, the value of weight a defined as:

$$
\alpha=\frac{A_{A}(x, y)}{A_{A}(x, y)+B_{A}(x, y)}
$$

In the eq. ( $26 \& 27)$, the larger the value of coefficients in a low frequency of two images with different focused objects is played a more important role in the fusion coefficient. Finally, valuable and pertinent information gains in the fusion image.

\subsubsection{The improved algorithm for the coefficient in high frequency}

The wavelet transformation coefficients gained the details change, which responses to information of the pixel in the original images, in the image in three directions-horizontal, perpendicular and diagonal. Therefore, the region with sharp changes in high-frequency corresponding to sufficient image detail information reflects the clarity of the source image. The fused image introduces the image clarity and the contrast factor in the wavelet transform domain. The proposed algorithm made visible progress on the decomposition process. Classing the coefficients into blocks in high frequency avoids the disturber of the noise. At the same time, the weight for blocks constructs to dismiss the block effects.

\section{Definition 3: The evaluation function for the image clarity}

Among the methods for assessing the image clarity, variance, and entropy, are the best because of the better performance of spatial parameters. However, the proposed measuring metrics are immune to the clarity changes of the source image, and it introduces the Edge Average acquaintance as the factor for the evaluation function of image coefficients in high frequency in which is defined as

$$
\begin{aligned}
\operatorname{Ctr}_{l}(i, j) & =\sum_{k=1}^{s}\left|d D_{l}^{\xi} / d x\right| \\
& \approx\left|D_{l}^{\xi}(i, j)-D_{l}^{\xi}(i, j-1)+\right| D_{l}^{\xi}(i, j)-D_{l}^{\xi}(i, j+1) \mid \\
& +\left|D_{l}^{\xi}(i, j)-D_{l}^{\xi}(i-1, j)+\right| D_{l}^{\xi}(i, j)-D_{l}^{\xi}(i+1, j) \mid \\
& +\left|\frac{D_{l}^{\xi}(i, j)-D_{l}^{\xi}(i-1, j-1)}{\sqrt{2}}\right|+\left|\frac{D_{l}^{\xi}(i, j)-D_{l}^{\xi}(i+1, j-1)}{\sqrt{2}}\right| \\
& +\left|\frac{D_{l}^{\xi}(i, j)-D_{l}^{\xi}(i-1, j+1)}{\sqrt{2}}\right|+\left|\frac{D_{l}^{\xi}(i, j)-D_{l}^{\xi}(i-1, j+1)}{\sqrt{2}}\right|
\end{aligned}
$$

Where $\operatorname{Ctr}_{l}(i, j)$ is the contrast value of coefficients in high frequency, which locals $(\mathrm{i}, \mathrm{j})$, and $D_{l}^{\zeta}(i, j)$ is the 
coefficients of high frequency in $l$ level and $\zeta$ direction ( $\zeta=\mathrm{H}, \mathrm{V}, \mathrm{D})$. Eq.28) is regarded in the sum of the weighted distance between each coefficient and its 8 neighbors. The weight for each coefficient neighbor is 1 in $0^{\circ}$ and $90^{\circ}$ direction and $1 / \sqrt{2}$ at $45^{\circ}, 135^{\circ}$ direction. The evaluation function is the extension statistics for each coefficient with its neighbors in the image. The sharper is with a large number reflects the clearer source image, so this function has an advantage in changing trends and high sensitivity.

\section{Algorithm 2: Algorithm for contrast based on a region}

One pixel of wavelet transforms coefficients is sensitive to the noise, and it cannot reflect the clearer image with a higher value. To overcome the disturber of the noise in the fusion process, the algorithm for contrast based on the region of the mutinous image fusion is handled as (i) grouping the wavelet transforms coefficients at each scale and each direction of source images in high frequency into smaller blocks. (ii) Compute the statistical region contrast value for the block based on the following descriptions.

1. Divide the wavelet transformation coefficient vectors into blocks in the level $l$ and direction $\zeta$ ( $\zeta=\mathrm{H}$,

$\mathrm{V}, \mathrm{D})$ into blocks with a size such as $3 \times 3,5 \times 5,8 \times 8$, and more. However, it will affect the quality of the result.

2. The value of the region contrast is computed based on eq.29.

$$
\begin{aligned}
\text { BlockCtr }{ }_{l}^{\zeta}(i, j)= & \sum_{\substack{m=1 n=1\\
}}^{M} \operatorname{Ctr}_{l}^{\zeta}((i-1) * M \\
& +m,(j-1) * N+n),\{i \in I, j \in J\}
\end{aligned}
$$

Where $I, J$ is the number of rows and columns for dividing the coefficient vector by the size $\mathrm{M} \times \mathrm{N} .(i, j)$ is the number of the block in the coefficient vector, and the value must be between 0 and $0-J$. The BlockCtr $\zeta_{l}(i, j)$ is the region's acutance in $l$ the level and $\zeta$ direction of the block $(i, j)$ in high frequency.

3. Construct the matching degree $\rho_{l}^{\zeta}(i, j)$ as equation (30).

$$
\rho_{l}^{\zeta}(i, j)=\frac{2 \times\left(\text { BlockCtr } \zeta_{A}^{\zeta}(i, j) \times \text { BlockCtr } \zeta_{B}^{\zeta}(i, j)\right)}{\left(\text { BlockCtr } \zeta_{A}^{\zeta}(i, j)\right)^{2}+\left(\text { BlockCtr } \mathrm{r}_{l}^{\zeta}(i, j)\right)^{2}}
$$

4. The computer threshold is between $0.5 \sim 0.8$, the optimal value of which depends on the source images. If $\rho_{l}^{\zeta}(i, j) \leq \alpha$ or smaller, then the combined coefficient vector of the block in $l$ level and $\zeta$ direction in high frequency in the image can be selected for the two source images like the eq. 31 else like eq. 32 .

$$
\begin{aligned}
& \left\{\begin{array}{l}
\text { if (BlockCtr } \left.l_{A}^{\zeta}(i, j) \geq \text { BlockCtr } l_{B}^{\zeta}(i, j)\right) \\
D_{l_{F}}^{\zeta}(i, j)=D_{l_{A}}^{\zeta}(i, j) \\
\text { else } \\
D_{l_{F}^{\zeta}}^{\zeta}(i, j)=D_{l_{B}}^{\zeta}(i, j)
\end{array}\right. \\
& \text { if (BlockCtr } \left.\zeta_{A}^{\zeta}(i, j) \geq \text { BlockCtr } l_{B}^{\zeta}(i, j)\right) \\
& D_{l_{F}}^{\zeta}(i, j)=D_{l}^{\zeta}(i, j) \times W_{l}^{\zeta} \max +D_{l}^{\zeta}{ }_{B}(i, j) \times W_{l}^{\zeta} \min \\
& \text { else } \\
& D_{l}^{\zeta}{ }_{F}(i, j)=D_{l}^{\zeta}(i, j) \times W_{l}^{\zeta} \min +D_{l}^{\zeta}{ }_{B}(i, j) \times W_{l}^{\zeta} \max
\end{aligned}
$$


Where $W_{l}^{\zeta} \max W_{l}^{\zeta}$ m in is computed by eq.33:

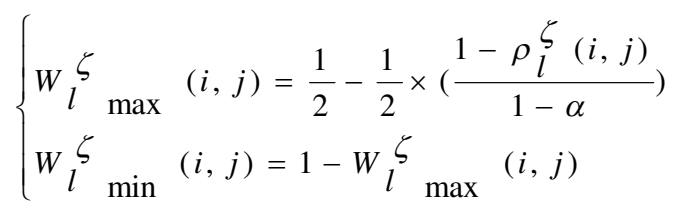

5. Validate consistency on the fusion coefficient.

If the coefficient block of the resulting image in high frequency comes from source image A, while its neighbors all come from source image $\mathrm{B}$, then it must be rewritten as the corresponding block of $\mathrm{B}$.

6. Dismiss the block effect.

If the two blocks that neighbor each other come from A and B, they will not agree with each other, called the block effect between the edges of the two blocks. To solve this problem, sum the value on the two source images with eq.32 and weights as eq. 33 .

$$
\left\{\begin{array}{l}
w_{l}^{\zeta} \max \\
w_{l}^{\zeta} \min
\end{array}(i, j)=\max \left(\rho_{l}^{\zeta}, 1-\rho_{l}^{\zeta}\right)\right.
$$

\section{Experiment 3:}

In this experiment, two source images, with differently focused, and the reference images used, as shown in Figure 7(a)-(c). The wavelet basis adopted is bior2; the number of decomposition levels is five; the block size in the algorithm is $5 \times 5$; the threshold is 7.6. Other algorithms implemented, for example, on local energy (Chang, 2012) and local variance (Nasrin et al., 2014), have not addressed the maximum of the wavelet transformation coefficient. The fusion images with various algorithms and the quality measurements showed in Figure 7 and Table 4.

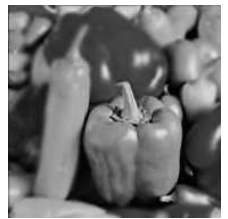

(a) The peppers focused on the front

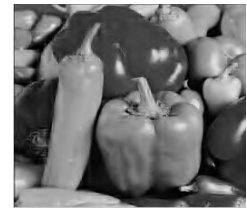

(d) Image with Max-value fusion

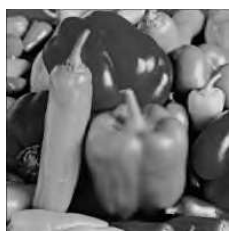

(b) The peppers focused on the background

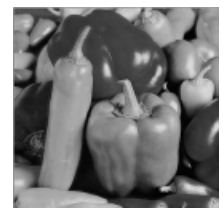

(e) image with part energy fusion

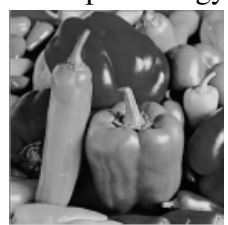

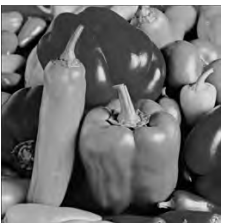

(c) The referenced image

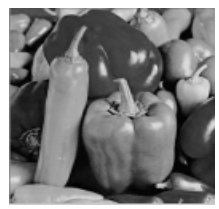

(f) image with local variance fusion

(g) Image with multi-focus with wavelet transform fusion (methods suggested in this paper)

Figure7 Fused images based on several methods 
Table 4 fusions result from different methods

\begin{tabular}{ccccccc}
\hline Algorithm & Entropy & $\begin{array}{c}\text { Standard } \\
\text { Deviation }\end{array}$ & Clarity & RMSE & $\begin{array}{c}\text { Mutual } \\
\text { Information }\end{array}$ & Joint Entropy \\
\hline $\begin{array}{c}\text { Absolute Value } \\
\text { Max }\end{array}$ & 7.5877 & 55.1146 & 6.9532 & 9.0230 & 0.6082 & $\mathbf{2 2 . 7 7 9 9}$ \\
$\begin{array}{c}\text { Local Energy Max } \\
\text { Local Variance }\end{array}$ & 7.5883 & 56.5355 & 7.9492 & 2.7974 & 0.6784 & $\mathbf{2 2 . 7 9 6 7}$ \\
$\begin{array}{c}\text { Method in this } \\
\text { paper }\end{array}$ & 7.5617 & 55.0692 & 7.0604 & 5.6828 & 0.6549 & $\mathbf{2 2 . 7 1 2 1}$ \\
\hline
\end{tabular}

Table 4 is the fusion images measuring summary value for more similar to the reference image, which indicates the informative parts of the source images with less RMSE value. The results of mutual information and RMSE are relatively the same.

\subsubsection{Result and discussion}

In this paper, the proposed integrated techniques for multi-focus image fusion experiments are analyzed in-depth on three different approaches to enhance fused image information quality. The testing experiments perform in pixelbased (table-2), decomposition level (table-3), and by methods (table-4). In the pix-based experiment, the image reality is enhanced and measured using various computational metrics, including (cross) entropy, mean square error, quality index, Peak Signal to Noise Ratio, and others in a different wavelet base. These measuring techniques revealed the structure of fused image information and its quality to localized the object. The fused image quality test and amplification of the results are shown in Table 2, which gives easy observation about the image fusion. The experiment results indicated that the proposed integrated technique is capable to exploit more image information and feature extraction ability

The objects or images better visual quality tested result presented in Table 3 and 4 by considering various image decomposition levels and methods. In this case, the computation results of entropy and cross-entropy revealed an enhanced information quality. The fusion image measuring value is more similar to the reference image. It indicates the informative parts of the source images with less RMSE value. The larger the entropy of fusion and mutual information is, the better fusion results. In addition, the smaller the cross-entropy gives a clear insight of more collaboration or relations between images and reflects minor differences of details and the features of textual changes.

\section{Conclusion}

Image data information quality is essential for various applications and medications. In this paper, I presented the integrity of DWT and CV-based multi-focus image fusion to improve the quality of fused images. In this critical analysis, the challenges of denoised low pass subband image data in-depth analysis of multi-focus image fusion. The integrity of DWT and CV is a novel technique to augment wavelet transform performance to enhance multi-focus image fusion information quality. This research considered various wavelet transformations to address and demystify the features' extractions level and selection accuracy for a better-fused image. The results are tested using different metrics techniques. The experiments revealed the efficiency of the algorithms to determine the best number of levels with in-depth feature extraction techniques. The proposed approach introduced the integrity of DWT and $\mathrm{CV}$ to minimize the challenges of image fusion complexity. It noted that the integrity of DWT and CV-based multifocus image fusion is a solution to optimize feature extraction time efficiency for high levels of noise image fusion. It is also an advanced technique to explore the object features in a selected region of the given image sources. Therefore, the performance of DWT is enhanced with computer vision. It is an advance and pertinent to enhance the wavelet coefficients in low-frequency based on the weight of each pixel, and high-frequency based on region 
contrast indicates the feasibility of the fused image quality and functionality. The essence of fusion image quality from video image is essential and extended research work.

\section{Acknowledgments}

I would like to thank the anonymous reviewers for their detailed review, valuable comments, and constructive suggestions. This research did not receive any specific grant from funding agencies in the public, commercial, or not-for-profit sectors.

\section{Reference}

[1] Agarwal Ruchi Sanjay et al., (2017), CT and MRI Image Fusion Based on Discrete Wavelet Transform and Type-2 Fuzzy Logic, International Journal of Intelligent Engineering and Systems, Vol.10, No.3, pp. 355-362,

[2] Aggarwal J. (1993) Multisensor Fusion for Computer Vision, Springer-Verlag,

[3] Andrey Osipov et al., (2018) Some Fuzzy Tools for Evaluation of Computer Vision Algorithms, International Journal of Computer Vision and Image Processing, Vol. 8, Issue 1, PP. 1-14,

[4] Ahmed F. Fadhil et al., (2019), Fusion of Enhanced and Synthetic Vision System Images for Runway and Horizon Detection, MDPI, Sensor, 3802; doi:10.3390/s19173802, pp. 1-17,

[5] Alberto Pretto, et al., (2010), Image similarity based on Discrete Wavelet Transform for robots with low-computational resources, Elsevier, Robotics and Autonomous Systems 58, pp. 879_888,

[6] Blasch, E.; Xiaokun Li; Genshe Chen; Wenhua Li (2008) Information Fusion, 11th International Conference on Digital Object Identifier: 10.1109/ICIF.2008.4632263 1-6,

[7] Cai Xiaohao et al., (2020), Wavelet-based segmentation on the sphere, Elsevier, Pattern Recognition, 100, pp. 1-15,

[8] Chang Ko-Chin, (2012), Multi-focus Image Fusion Using Local Energy Pattern, Applied Mechanics and Materials Vol 145, pp 119-123,

[9] Chen Wei-bin et al., (2019), Fusion Algorithm of Multi-focus Images with Weighted Ratios and Weighted GradientBased on Wavelet Transform, J. Intell. Syst., 28(4): pp. 505-516,

[10] David AY (1995) Image Merging and Data Fusion using the Discrete Two-Dimensional Wavelet Transform [J], J. Opt. Soc. A, 12(9):1834-1841.

[11] De, I., Chanda, B. (2006) A simple and efficient algorithm for multi-focus image fusion using morphological wavelets, Signal Process. 86 (5), 924-936,

[12] Deepika. L and Mary Sindhuja. N.M, (2014), Performance Analysis of Image Fusion Algorithms using HAAR Wavelet, IJCSMC, Vol. 3, Issue. 1, pg.487 - 494,

[13] Devyani P. Deshmukh and Malviya A. V., (2015) A Review on Image Fusion using Wavelet Transform, International Journal of Engineering Trends and Technology (IJETT) - Vol. 21, No. 8, pp. 376-279,

[14] Dhirendra Mishra and Bhakti Palkar, (2015), Image Fusion Techniques: A Review, International Journal of Computer Applications, (Vo. 130, No.9, pp. 7-14,

[15] Fauhan Handay Pugar and Aniati Mumi Arymurthy, (2019), Blind Color Image Watermarking Based on 2-level Discrete Wavelet Transform, Mary Modulation, and Logistic Map, IEEE, $12^{\text {th }}$ International Conference on Information \& Communication Technology and System, pp. 235-240,

[16] Harpreet Kaur and Rachna Rajput, (2015), A Combined Approach using DWT \& PCA on Image Fusion, International Journal of Advanced Research in Computer and Communication Engineering Vol. 4, Issue 9, pp. 294-296,

[17] Kavitha S. and Thyagharajan K. K., (2017) "Efficient DWT-based fusion techniques using genetic algorithm for optimal parameter estimation," Soft Computing., vol. 21, no. 12, pp. 3307-3316,

[18] Kulvir Singh and Neeraj Julka, (2016), Image Fusion Methodology Using Hybrid Pyramidal DWT-Lp Approach International Journal of Advanced Engineering and Research, Development Vol. 3, Issue 1, pg. 263-268,

[19] Li ChangSheng et al., (2018), Multi-focus image fusion method for image acquisition of 3D objects, Applied Optics, Vol. 57, No. 16, pp. 4514-4523,

[20] Li Huafeng et al., (2018) Joint medical image fusion, denoising and enhancement via Discriminative low-rank sparse dictionaries learning, Elsevier, Pattern Recognition, 79, pp. 130-146,

[21] Li Jinjiang et al., (2019), Multifocus Image Fusion Using Wavelet-Domain-Based Deep CNN, Hindawi, Computational Intelligence and Neuroscience, Vol. 2019, pp. 24-48,

[22] Li X., He M., and Rou M., (2010), Multi-focus image fusion based on redundant wavelet transform, IET Image Process., 2010, Vol. 4, Iss. 4, pp. 283-293, 
[23] Li Yibao et al., (2020) Multi-component volume reconstruction from slice data using a modified multi-component CahnHilliard system, Elsevier, Pattern Recognition, 93, pp. 124-133,

[24] Lu Tang et al., (2017) Multimodal Medical Image Fusion Based on Discrete Tchebichef Moments and Pulse Coupled Neural Network, Wiley Periodicals, Inc., Vol. 27, pp., 57-65,

[25] Ma Jizhou et al., (2020) Adaptive appearance modeling via hierarchical entropy analysis over multi-type, features, Elsevier, Pattern Recognition, 98, 1-14,

[26] Mallat S G (1989) A theory for Multi-resolution Signal Decomposition: The Wavelet Representation [J]. IEEE Trans. On Pattern Anal. Mach. Intel, 11(3): 674-693.

[27] Manu V T and Philomina Simon, (2012), A Novel Statistical Fusion Rule for Image Fusion and its Comparison in NonSub Sampled Contourlet Transform Domain and Wavelet Domain, The International Journal of Multimedia \& Its Applications (IJMA) Vol.4, No.2, pp. 69-87,

[28] Maria C. Mariani et al., (2020) Analysis of stock market data by using Dynamic Fourier and Wavelets techniques, Elsevier, Physic A, PP. 1-13,

[29] Maruthi R. and Lakshmi I., (2017), Multi-Focus Image Fusion Methods - A Survey, IOSR Journal of Computer Engineering (IOSR-JCE, Vol.9, Issue 4, pp. 9-31,

[30] Meenu Manchanda and Deepak Gambhir, (2019), Multi-focus image fusion based on wave atom transform, Sådhanå, Indian Academy of Sciences, pp. 1-16,

[31] Meenu Manchanda and Rajiv Sharma, (2017) Multifocus Image Fusion Based on Discrete Fuzzy Transform, the IEEE WiSPNET 2017 conference, pp. 775-779,

[32] Nada Habeeb et al., (2015), Multi-Sensor Fusion based on DWT, Fuzzy Histogram Equalization for Video Sequence, The International Arab Journal of Information Technology, Vol. 15, No. 5, Pg. 825-830),

[33] Nasrin Amini et al., (2014), MRI-PET image fusion based on NSCT transform using local energy and local variance fusion rules, J Med Eng Technol, 38(4): pp. 211-219,

[34] Natchammai L A and Hariharan K., (2019), Image Enhancement with Medical Image Fusion Based ISH, International Journal of Innovative Technology and Exploring Engineering, Vol. 8, Issue 6, Pg. 649-653,

[35] Nianyi Wang et al., (2015), Multi-Focus Image Fusion Based on Nonsubsampled Contourlet Transform and Spiking Cortical Model, CTU FTS, pp. 623-639,

[36] Nikita D.Rane et al., (2017), Comparative study of Image Fusion Methods: A Review, International Journal of Engineering and Applied Sciences (IJEAS), Vol. 4, Issue.10, pp. 67-72

[37] Pajares, G., Cruz, J.M. (2004) A wavelet-based image fusion tutorial, Pattern Recognition 37 (9), 1855-1872,

[38] Qiang Zhang et al., (2020) Multi-focus image fusion based on non-negative sparse representation and patch-level consistency rectification, Elsevier, Pattern Recognition, 104, pp. 1-14,

[39] Qiang Wang et al., (2019) Laplacian pyramid adversarial network for face completion, Elsevier, Pattern Recognition, 88, pp. 493-505,

[40] Radhika Vadhi et al., (2017), ICMAEM: IOP Conf. Series: Materials Science and Engineering, 225 012156, pp. 1-14,

[41] Rajesh Bhandari and Shivakumar B. R, (2016), Wavelet-based Analysis of Medical Image Fusion using MATLAB GUI, International Journal of Innovative Research in Science, Engineering and Technology, Vol. 5, S. Issue 9, pp. 512-517,

[42] Roosta I. et al., (2015), Multi-focus Image Fusion Based on Surface Area Analysis, 978-1-4799-8339-1/15/\$31.00 IEEE, pp. 2805-2809,

[43] Sankaran K. Sakthidasan alias and Nagarajan V., (2019), Noise Removal Through the Exploration of Subjective and Apparent Denoised Patches Using Discrete Wavelet Transform, Taylor and Francis, IETE Journal of research, pp. 1-10,

[44] Sarwar Shah Khan et al., (2021), Hybrid Sharpening Transformation Approach for Multifocus Image Fusion Using Medical and Nonmedical Images Research Article, Hindawi, Journal of Healthcare Engineering Volume 2021, Article ID 7000991, 17 pages doi.org/10.1155/2021/7000991,

[45] Sebastian Zambanini (2019), Feature-based Groupwise registration of historical aerial images to present-day orthophoto maps, Elsevier, Pattern Recognition, 99, pp. 66-77,

[46] Surabhi Agarwal and Sameeksha Chaudhary, (2018) High PSNR based Image Fusion by use Brovey Transform, International Journal of Engineering Development and Research, Vol. 6, Issue 1, pp. 415_461,

[47] Suthakar R. Johnson et al., (2014), Study of Image Fusion- Techniques, Method and Applications, IJCSMC, Vol. 3, Issue. 11, pg. $469-476$,

[48] Tao Wan et al., (2013), Multifocus image fusion based on robust principal component analysis, Pattern Recognition Letters 34, pp., 1001-1008,

[49] Toet, A. (1989.) Image fusion by a ratio of the low-pass pyramid. Pattern Recognition Lett. 9 (4), $245-253$.

[50] Tian Lianfang et al., (2018), Multi Focus Image Fusion using Combined Median and Average Filter based Hybrid 
Stationary Wavelet Transform and Principal Component Analysis, International Journal of Advanced Computer Science and Applications, Vol. 9, No. 6, Pg. 34-41,

[51] Tejashri Polkamwar and Alpana Deshmukh, (2015), A Review on Multilevel Image Fusion Using Wavelet and Curvelet Transform, International Journal for Research in Applied Science \& Engineering Technology (IJRASET), Vol. 3, Issue VI, pp. 783-787,

[52] Ting Wu et al., (2019), An improved nondestructive measurement method for salmon freshness based on spectral and image information fusion, Elsevier, Computers, and Electronics in Agriculture 158, PP. 11-19

[53] Vaishnavi J. Deshpande and Jignyasa Sanghavi, (2019), Augmented Reality: Technology Merging Computer Vision and Image Processing by Experimental Techniques, International Journal of Innovative Technology and Exploring Engineering, Vol. 8, Issue. 8, pp. 534-537,

[54] Velliangiri S. (2019), Improved Security in Multimedia Video Surveillance Using 2D Discrete Wavelet Transforms and Encryption Framework, 3D Express, 10:17, PP. 1-9,

[55] Vivek Arya et al., (2019) An Efficient Adaptive Algorithm for Electron Microscopic Image Enhancement and Feature Extraction, International Journal of Computer Vision and Image Processing, Vol. 8, Issue 8, pp. 1-16,

[56] Wu-Chih Hu et al., (2012), Robust image watermarking based on discrete wavelet transform-discrete cosine transformsingular value decomposition, Journal of Electronic Imaging 21(3), pp. 1-8,

[57] Xiao Fuyuan, (2019), Multi-sensor data fusion based on the belief divergence measure of evidence and the belief entropy, Elsevier, Information Fusion, Vol. 46, pp. 23-32,

[58] Xueping Dai et al., (2019), New method for denoising borehole transient electromagnetic data with discrete wavelet transform, Elsevier, Journal of Applied Geophysics 168, PP. 41-48,

[59] Yang, Y., Huang, S., Gao, J., \& Qian, Z. (2014). Multi-focus image fusion using an effective discrete wavelet transformbased algorithm. Measurement science review, 14(2), 102,

[60] Yi Chai et al., (2012), a Multifocus image fusion based on features contrast of multiscale products in the nonsubsampled contourlet transform domain, Elsevier, Optik 123, pp. 569-581,

[61] Yihang Zhang et al, (2018), Spatial-temporal fraction map fusion with multi-scale remotely sensed images, Elsevier, Remote Sensing of Environment 213, pp. 162-181

[62] Yogita Jawale and Andurkar A. G., (2013), Implementation of Image Fusion Technique Using Wavelet Transform, International Journal of Science, Engineering and Technology Research (IJSETR) Volume 2, Issue 3, pp. 695-697,

[63] Yong Yang et al., (2014), Effective Multi-Focus Image Fusion Based on HVS and BP Neural Network, Hindawi, Scientific World Journal, Vol. 2014, pp. 1-10,

[64] Yushu Liu et al., (2013), Multi-focus Image Fusion Based on Multiresolution Transform and Particle Swarm Optimization, Advanced Materials Research Vols 756-759 (2013) pp 3281-3285,

[65] Zhan Lingchao et al., (2017), Infrared and Visible Images Fusion Method Based on Discrete Wavelet Transform, Journal of Computers Vol. 28, No. 2, pp. 57-71,

[66] Zhang, Z., Blum, R.S., (1999) A categorization of multiscale-decomposition-based image fusion schemes with a performance study for a digital camera application, Proc. IEEE 87 (8), 1315-1326.

[67] Zhao Xuemei et al., (2020) Remote sensing image segmentation using geodesic-kernel functions and multi-feature spaces, Elsevier, Pattern Recognition, 104, pp. 1-14,

[68] Zheng Kangfeng and Wang Xiujuan, (2018) Feature selection method with joint maximal information entropy Between features and class, Elsevier, Pattern Recognition, 77, PP. 20-29,

Dr. Gebeyehu Belay Gebremeskel is an associate professor at Bahir Dar University, Ethiopia. He did his post-doctoral research at Chongqing University, China. He received his Ph.D. from Chongqing University, in July 2013, a Master's Degree from London South Bank University, UK, and B. Sc. Degree from Alemaya University, Ethiopia. Dr. Gebeyehu gained solid experience in research and teaching in different positions and institutes. He also engaged and contributed fundamental professional skills in various IT projects and research activities. His research interest includes Machine Learning/Data Mining, Big Data Analytics, Smart Environment, Agent Technologies, Artificial Intelligence, Business Intelligence, Data cloud, and others in the field of Computer Science and Engineering. Currently, he is working at Bahir Dar University, at Bahir Dar Institute of Technology. He is advising Masters and Ph.D. students. Besides this, he is also actively participating in the Institute affairs in a different position. At present, he is head of the Software Engineering Department, and chair for system development. 